\title{
Uso del portafolio en la formación preclínica del graduando en odontología
}

\author{
Claudio M. Guerra
}

Introducción. Las escuelas de medicina del mundo están reformulando sus estructuras curriculares, contenidos y estrategias para integrar las tendencias actuales de la educación superior. Esto implicará un cambio en la evaluación: las técnicas tradicionales ya no serán congruentes con los nuevos modelos curriculares, si se requiere una contribución al aprendizaje. La bibliografía señala al portafolio como una herramienta que permite la evaluación del desempeño y estimula el aprendizaje. Su uso en la educación médica de pregrado no se ha explorado extensamente y se halla poca evidencia sobre su aplicación específica en odontología.

Materiales y métodos. Bajo una investigación educativa de acción, se puso en práctica en la asignatura 'Prostodoncia I', del segundo año de la carrera de odontología. La utilización de esta herramienta tuvo como objetivo que docentes y alumnos se conciencien sobre la responsabilidad compartida de enseñar y aprender valorando las potencialidades de la implementación del portafolio. Se realizó a partir de reuniones docentes, entrevistas y encuestas a los alumnos.

Resultados. La opinión de los estudiantes en las encuestas expresa que el portafolio mejoró su aprendizaje significativamente y fue una buena herramienta de enseñanza, contribuyendo a la reflexión en su trabajo académico. Los profesores reconocieron la importancia de un espacio para el acercamiento al alumno, permitiendo realizar un análisis de sus producciones y su relación con los contenidos de la asignatura.

Conclusiones. Se demostró que el portafolio se considera una herramienta que permite enriquecer las prácticas educativas, generando conocimientos desde las construcciones de los alumnos y las intervenciones de los profesores.

Palabras clave. Aprendizaje reflexivo. Autoevaluación. Enseñanza reflexiva. Evaluación formativa. Odontología. Portafolio.

\section{Use of the portfolio in the students dental preclinical training}

Introduction. The schools of medicine in the world are reformulating their contents, strategies and curricular structures to integrate current trends in higher education. This will involve a change in the evaluation: traditional techniques are no longer consistent with the new curriculum models, if required a contribution to learning. The literature points to the portfolio as a tool for performance evaluation and stimulates learning. Its use in undergraduate medical education has not been explored extensively, finding little evidence of their specific application in dentistry.

Materials and methods. It was decided its application in coincidence with the educational objectives of a course from the second year of the career of dentistry. The use of this tool, as a subject in the curriculum of the career of dentistry has the purpose that teachers and pupils get conscience about responsibility they must shake learning and teaching but giving value to the potentialities in the implementation of portfolio. It was done since meetings with teachers, interviews and surveys to the pupils.

Results. student opinion surveys state that the portfolio significantly improved their learning and it was a good teaching tool, helping to reflect on their academic work. The teachers recognized the importance of space for student-centered approach, allowing analysis of its productions and its relation to the contents of the subject.

Conclusions. After analyzing the results, it was demonstrated that portfolio is considered a tool that let enrich educational practices, creating new knowledge since pupils' constructions and teachers' participations.

Key words. Dentistry. Formative evaluation. Portfolio. Reflective learning. Reflective teaching. Self assessment.
Escuela de Odontología. Universidad del Salvador. Buenos Aires, Argentina.

Correspondencia: Dr. Claudio Miguel Guerra. Escuela de Odontología. Universidad del Salvador. Junín, 959. Buenos Aires, Argentina.

E-mail: cmguerra@fibertel.com.ar

Agradecimientos:

Al personal docente de la asignatura 'Prostodoncia I': R. Ruarte, A. Galbarini, J. Panini, N. Bottazini, N. Politis, M. Minini, B. Caffaro Freire y J. Dervissouglu. A R. Beltrán, R. Modlin y B. Fernández, por sus oportunas correcciones.

Conflicto de intereses: No declarado.

Competing interests: None declared.

(c) 2014 FEM 


\section{Introducción}

En el mundo de la educación en el área de las ciencias de la salud, son múltiples las propuestas para explorar nuevas alternativas de evaluación de a los estudiantes.

El portafolio es un instrumento originado dentro del campo de la educación artística y se considera un elemento innovador por su aplicación como estrategia evaluativa en diversos contextos académicos, constituyendo una herramienta funcional para la construcción de conocimiento. En este sentido, los portafolios pueden utilizarse con el fin de mejorar la práctica de las ciencias médicas en general, identificando a profesionales con bajo desempeño y reforzando los procesos de aprendizaje [1].

El portafolio se ha manifestado, en general, sólo como una herramienta para evaluar el aprendizaje de los alumnos, pero su uso no puede restringirse sólo a eso. El portafolio es una estrategia de enseñanza para los docentes y también una estrategia de aprendizaje para los alumnos, poniendo en juego múltiples factores y variables que inciden en el proceso educativo.

Se convierte así en una herramienta que permite al docente reflexionar sobre sus propuestas de enseñanza a partir de la evaluación continua de éstas y de los resultados de aprendizaje de sus alumnos. Esto significa también una transformación del papel del docente, una nueva definición de los objetivos, un cambio en el enfoque de las actividades educativas y un cambio en la direccionalidad del aprendizaje.

En este sentido, también el alumno puede reflexionar y evaluar su propio trayecto guiado por el docente, que lo acompaña durante todo el proceso, reconociendo su protagonismo en el aprendizaje y favoreciendo la autoevaluación a partir de la toma de conciencia de las fortalezas y debilidades, avances y retrocesos por los que se atraviesa.

Lejos de ser una evaluación tradicional, el portafolio favorece la metacognición y se materializa en evidencias que permiten la reflexión sobre la acción [2]. El análisis de situaciones críticas permiten cuestionar lo conocido y complejizar el pensamiento y la construcción de nuevos conocimientos.

El portafolio es un instrumento que se originó dentro de la educación artística y por su aplicación a otros ámbitos educativos se considera un elemento innovador, que comenzó a extender su uso como estrategia evaluativa y como herramienta para atender los procesos de construcción del conocimiento.

Hay coincidencia en la revisión bibliográfica en que el portafolio constituye una herramienta de evaluación que estimula el aprendizaje y permite la evaluación del desempeño. Se emplea ampliamente en diversos contextos académicos. Su uso en la educación médica de pregrado no se ha explorado extensamente y no se ha encontrado referencia alguna sobre su aplicación específica en odontología.

Numerosos autores como Barberà [3], Klenowski [4], Barragán [5] y Villalobos [6], entre otros, señalan el potencial de esta herramienta para estimular la autonomía del estudiante en la construcción del conocimiento, el pensamiento reflexivo, la autoevaluación y la metacognición.

En experiencias previas, en relación al uso del portafolio en educación, reconocidas universidades han realizado investigaciones demostrando un alto grado de satisfacción del portafolio como herramienta para la evaluación y autoevaluación [5,7-9].

En la Universidad Nacional Autónoma de México (UNAM), el uso del portafolio convencional se ha implementado en la enseñanza de las ciencias básicas y clínicas, en la formación docente y en la formación académica de los alumnos. Recientemente se implementó el e-portafolio. Esta experiencia, al trabajar en el portafolio con los alumnos y los docentes, intenta promover más espacios de comunicación y gestionar los contenidos médicos [10].

En Argentina se realizó una búsqueda de antecedentes acerca de la implementación del portafolio en otras facultades de odontología del país, además de una revisión bibliográfica. Sin embargo, no se encontró una experiencia similar.

Teniendo en cuenta que la investigación en educación tiene como propósito principal la reflexión y mejora de las tareas docentes, el objetivo de la experiencia expuesta en el presente artículo consistió en develar si el portafolio resultaba beneficioso para el trinomio enseñanza-aprendizaje-evaluación en alumnos que se preparaban para su inicio en la práctica clínica odontológica, con especial interés en el capítulo de la rehabilitación oral por conjugar aspectos del conocimiento, de las destrezas y de las actitudes. De este modo, se apuntó a valorar su utilidad como instrumento de evaluación formativa para el aprendizaje de los alumnos y la ponderación de los docentes. Asimismo, la investigación se propuso promover y activar los procesos de reflexión en el plantel docente y la autoevaluación de los alumnos.

\section{Materiales y métodos}

El estudio se inició durante el año lectivo 2009, cuando comenzó a planificarse el uso del portafolio en la asignatura. Para ello se realizaron, durante ese año, reuniones docentes que definieron su puesta en mar- 
cha y se acordaron los criterios y objetivos del estudio que se realizaría en el marco de su implementación.

La muestra seleccionada para llevar a cabo el estudio estuvo constituida por 72 estudiantes que, durante los años académicos $2010(n=38)$ y 2011 $(n=34)$, cursaron la materia 'Prostodoncia I' del segundo año de la carrera de odontología de la Universidad del Salvador -en convenio de colaboración con la Asociación Odontológica Argentina, en Buenos Aires-. Asimismo, intervinieron ocho docentes de distinta jerarquía: un profesor titular, un profesor asociado, cuatro jefes encargados de grupos de trabajos prácticos y dos docentes auxiliares. Todos tuvieron una participación activa en el desarrollo de los contenidos y prácticas de la asignatura, en contacto directo con los alumnos.

Con la intención de valorar las potencialidades de la implementación del portafolio, analizando su compatibilidad con las prácticas establecidas en cuanto a la formación del alumnado para la clínica de la rehabilitación oral a través de la prostodoncia, en 2009 se definieron los criterios que organizarían la implementación de la propuesta (Tabla I).

\section{Generación de los documentos del portafolio}

En el marco de las reuniones con los profesores, y a partir de la búsqueda bibliográfica realizada, se definieron los criterios que deberían reunir los portafolios presentados por los alumnos. Se analizaron las condiciones y cantidad de documentos, siguiendo en ese sentido ciertas recomendaciones acerca de que su número no debía ser excesivo. Se consensuó que cada portafolio debía reflejar el proceso vivido por el alumno. Las condiciones y formas de presentación de los documentos requeridas a los alumnos fueron las siguientes:

\section{Identificación}

Los documentos debían presentarse en tamaño de hoja A4 y ubicar la identificación del alumno en el margen superior derecho para reconocer rápidamente a quién correspondía la carpeta presentada. Por otro lado, es importante que el alumno pueda establecer una estructura y organización internas para su propio portafolio elaborando una tabla de contenidos que incluya índice, contenidos, objetivos, tareas, etc.

\section{Extensión de los trabajos}

Las respuestas expresadas por escrito no debían superar las 50 palabras. Este criterio se basó en que la capacidad de síntesis supone un proceso cognitivo complementario del analítico que persigue una ma- yor comprensión de los hechos y fenómenos en su globalidad. Además, esta capacidad está íntimamente ligada con la capacidad de abstracción y de análisis, todas ellas competencias esperadas en alumnos universitarios [11]. Asimismo, se consideraría en la evaluación la ortografía por dar cuenta del grado de crecimiento académico en el ámbito universitario.

\section{Autoría de las producciones}

No se aceptarían trabajos realizados por terceros. Todos los esquemas o dibujos debían representar el trabajo personal de cada alumno, ser de autoría propia. En la vida universitaria no hay cursos de visualización, imaginación, creatividad o habilidades perceptivas; por ello hubo una decisión explícita de incorporar un profuso número de dibujos en el desarrollo de las consignas, al considerar su importancia de acuerdo con los criterios de Edwards [12] y McLean [13]. Según Edwards [12], 'las artes son imprescindibles para el aprendizaje de formas de pensamiento específico visual y perceptivo, igual que lo numérico y analítico. Ambos son vitales a la hora de adquirir el pensamiento crítico, de la extrapolación de los significados y la resolución de problemas. Deben aprovecharse las técnicas del arte para solucionar cuestiones académicas y otras de índole más cotidiana'.

\section{Objetivos de los trabajos presentados en el portafolio}

En cada documento, el alumno debía consignar cuál era, a su entender, la razón por la que incluía cada tema en el portafolio, indicando por qué lo consideraba parte de su aprendizaje.

El portafolio no debe ser una mera recopilación de asignaciones, sino que los trabajos expuestos deben mostrar evidencias de los procesos de aprendizaje personal, metacognitivos y afectivos. Por razones formativas y evaluativas, pueden establecerse algunos requisitos mínimos de los trabajos que deben incluirse en el portafolio, pero debe privilegiarse la participación autónoma del estudiante en la selección de los materiales que desea incluir como muestra de sus procesos, sus progresos y dificultades.

\section{Plan de acción}

El alumno debía narrar los inconvenientes y problemas surgidos en el desarrollo del documento o en la acción práctica correspondiente, señalando asimismo las diversas propuestas de mejora para superar las dificultades y los motivos de elección de alguna de ellas. El principal objetivo de esta propuesta era la reflexión sobre las evidencias recogidas y los aprendizajes adquiridos. 
Tabla I. Criterios para la implementación del portafolio (2009).

\begin{tabular}{|c|c|c|c|}
\hline \multirow{4}{*}{$\begin{array}{l}\text { Generación de } \\
\text { los documentos } \\
\text { del portafolio }\end{array}$} & Tipo de herramienta & \multicolumn{2}{|c|}{ Portafolio cerrado } \\
\hline & Duración & \multicolumn{2}{|c|}{ Un año. Ciclo lectivo correspondiente al cursado de la asignatura } \\
\hline & Elección de temas/actividades & \multicolumn{2}{|c|}{$\begin{array}{l}\text { Propuestas por los docentes de acuerdo con los objetivos de la asignatura, } \\
\text { focalizando la expresión a través del dibujo }\end{array}$} \\
\hline & Elaboración & \multicolumn{2}{|c|}{$\begin{array}{l}\text { Secuencial: de acuerdo al cronograma de temas y actividades de la asignatura } \\
\text { Parcial: entrega de borradores con tutorías docentes } \\
\text { Obligatoria }\end{array}$} \\
\hline \multirow{3}{*}{$\begin{array}{l}\text { Encuentros con } \\
\text { los estudiantes }\end{array}$} & Organización & \multicolumn{2}{|c|}{$\begin{array}{l}\text { Un encuentro al comienzo del ciclo lectivo para explicar las características del } \\
\text { portafolio y la modalidad de trabajo }\end{array}$} \\
\hline & Devolución & \multicolumn{2}{|c|}{ Tutorías docentes para el diseño del plan de acción } \\
\hline & Evaluación & \multicolumn{2}{|c|}{$\begin{array}{l}\text { Formativa: a través de tutorías y entregas parciales del portafolio } \\
\text { Evaluación final grupal de la experiencia }\end{array}$} \\
\hline \multirow{4}{*}{$\begin{array}{l}\text { Estrategias } \\
\text { de valoración }\end{array}$} & \multirow{2}{*}{ Opinión de los alumnos } & Herramienta & Encuesta cerrada, con algunas preguntas abiertas \\
\hline & & Participación & Voluntaria y anónima al finalizar el ciclo lectivo \\
\hline & \multirow{2}{*}{ Evaluación de los profesores } & Herramienta & Encuesta cerrada por escala tipo Likert \\
\hline & & Participación & Voluntaria de los docentes participantes de la experiencia \\
\hline
\end{tabular}

\section{Fuentes bibliográficas}

En cada documento se debía consignar la fuente bibliográfica del tema desarrollado. Las fuentes debían ser fiables y las citas debían realizarse correctamente. Este criterio se aplicó porque es sumamente importante para un alumno universitario reconocer la fiabilidad de la información y la mención de otros autores para información que no es de su autoría.

\section{Propiedad privada y} selección de experiencias

Los documentos que conformaban el portafolio y, por ende, el portafolio en sí, son propiedad de cada alumno. En cada actividad del curso, los alumnos debían facilitarlo a fin de ser consultado por sus compañeros y docentes. Hoy día hablamos de aprendizaje colaborativo: la colaboración favorece la producción de conocimientos y muchos de los avances de los alumnos se logran gracias a la socialización en los procesos de enseñanza y de aprendizaje. La interacción entre los alumnos y los profesores hacen muy diferente la dinámica de trabajo. Bajo estas consideraciones, cada alumno debía decidir de qué modo compartiría con el grupo aquellas experiencias que considerara importantes.

\section{Actividad de investigación}

Esta actividad fue opcional y representó la posibilidad de preparar un trabajo de investigación a partir de algunos de los temas trabajados en los portafolios. En ese marco, el tema era elegido por los alumnos, quienes debían obtener un corpus propio a partir de una fuente correcta y concreta. Los docentes cumplían el rol de tutores, facilitando las nociones iniciales sobre el manejo de pautas para la investigación, fuentes bibliográficas y conclusiones.

La incorporación de los alumnos en trabajos de investigación proporciona la posibilidad de consultar diferentes fuentes de información con el propósito de aumentar los conocimientos sobre un tema determinado. Es una de las competencias que se espera que los alumnos desarrollen durante su trayecto universitario porque favorece la evaluación crítica de la información y de sus fuentes y la aplicación de las consideraciones metodológicas.

\section{Encuentros con los estudiantes}

Para presentar la experiencia a los alumnos se realizó un encuentro inicial al comienzo de cada ciclo lectivo, 2010 y 2011, donde se explicaron las carac- 
Tabla II. Ventajas y desventajas del uso del portafolio.

1. ¿Qué ventajas crees que ha tenido trabajar con la metodología aportada por la guía-portafolio en comparación con la forma en que se hacía hasta ahora?

Preguntas de respuesta abierta

2. ¿Cuáles son los inconvenientes?

3. De cara al desarrollo futuro de esta herramienta docente, ¿qué sugerirías para que pueda mejorarse y ser más útil?

4. El uso del portafolio contribuyó a mi aprendizaje de forma significativa

5. Fue una buena herramienta de enseñanza

Preguntas de respuesta cerrada (medidas con la escala tipo Likert)

\section{El portafolio contribuyó a la reflexión de mi trabajo académico}

7. El portafolio contribuyó a un trabajo en equipo

8. Cualifica la enseñanza que se te ofreció en este curso anual de 'Prostodoncia I'

9. ¿En qué medida te sientes preparado para empezar la actividad clínica?
Tabla III. Opinión de los docentes colaboradores en relación a la experiencia.

Los documentos elaborados por el alumno fueron pruebas del proceso de aprendizaje

Del análisis de esas pruebas se pueden identificar los ciclos de aprendizaje, lo cual no ocurre con los métodos tradicionales

Permite al alumno ubicarse con facilidad en qué lugar del curso se encuentra

El alumno siente el aprendizaje como algo propio y de su responsabilidad porque puede decidir cuándo mejorar sus trabajos

Aparece un espacio de reflexión a través de la escritura

Permite integrar las tareas del proceso de aprendizaje con la evaluación

Se concibe como un tipo de evaluación alternativa que se basa en la negociación y en el intercambio comunicativo entre el profesor y el estudiante terísticas del portafolio y la modalidad de trabajo. Se les informó del objetivo del estudio y de que la evaluación sería sólo formativa respecto al desarrollo del portafolio a lo largo del año, a su vez que era indispensable contar con su opinión para el posterior análisis de esta experiencia.

En los períodos 2010 y 2011, las tutorías estuvieron a cargo de los jefes de grupo, a través de los 52 encuentros que comprendió el desarrollo de la asignatura. Cada jefe era responsable de un grupo, de hasta diez alumnos, teniendo contacto cotidiano con ellos al observar el desarrollo de sus destrezas y actitudes frente a la propuesta. Durante la actividad en el aula-taller se conformaron cuatro grupos de trabajos prácticos, de ocho alumnos cada uno.

Los encuentros tutoriales individuales se concertaron cuando los alumnos iban terminando la realización de los trabajos prácticos, según el cronograma para el desarrollo del ciclo lectivo. No se calificó la puntualidad en la entrega de los documentos ni el número de horas dedicadas para la realización del portafolio, dado que no era un requisito para la regularidad en la asignatura. Las tutorías se centraron en la explicación y fundamentación de los documentos elaborados por los alumnos.

Al finalizar la asignatura se llevó a cabo un encuentro de cierre donde prevaleció la intención de obtener la percepción de los alumnos acerca de cómo vivieron la experiencia de aplicación del instrumento.

\section{Estrategias de valoración}

Se realizaron dos estrategias para la valoración del estudio. Por un lado, se llevó a cabo una encuesta de opinión dirigida a los alumnos que cursaron la asignatura en cada ciclo lectivo. En segundo lugar, se realizó una encuesta cerrada de escala tipo Likert dirigida a los docentes.

\section{Opinión de los alumnos}

La encuesta dirigida a los alumnos fue de participación voluntaria y anónima al finalizar cada ciclo lectivo (2010 y 2011). La finalidad de esta encuesta fue recabar información acerca de la experiencia para comprender la concepción del instrumento didáctico desde la perspectiva de los estudiantes. Se obtuvo la participación de 53 alumnos: 23 del año académico 2010 y 28 del 2011.

En relación a la estructura de la encuesta, estuvo conformada por tres preguntas de respuesta abierta y seis preguntas de respuesta cerrada (cuatro sobre el portafolio y dos sobre la actividad docente desarrollada), medidas con la escala tipo Likert (1: muy de acuerdo; 2: de acuerdo; 3: neutral; 4: en desacuerdo; 5: muy en desacuerdo), y consistió en indagar sobre ventajas y desventajas de la aplicación del instrumento (Tabla II).

\section{Evaluación de los profesores}

Para recabar la opinión de los profesores colabora- 
Figura 1. Inconvenientes del uso del portafolio.

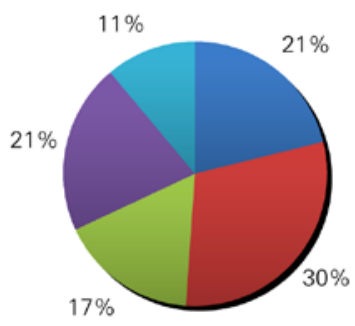

- Tiempo de trabajo

- Dificultades con el dibujo

- Trabajo con contenidos desconocidos

- No tuvieron

inconvenientes - Otros

dores en el desarrollo de la experiencia, se realizó una encuesta que constó de siete preguntas que debieron responderse también mediante una escala tipo Likert. Los ocho profesores manifestaron una coincidencia absoluta en todas las respuestas en el ítem 'muy de acuerdo' en relación al desarrollo de la experiencia (Tabla III).

En el grupo de discusión de profesores hubo coincidencia en que la experiencia reveló los procesos que, según Camillioni [14], requiere la evaluación formativa: la recogida de datos sobre el rendimiento, la observación del comportamiento del alumno mientras realiza su tarea y reflexiona, el registro de lo observado y la adaptación de las estrategias de enseñanza y aprendizaje según la información recogida y su interpretación por el profesor.

Se discutió y analizó si los beneficios e inconvenientes enunciados en la bibliografía coincidían con el parecer de los docentes partícipes. Se decidió utilizar como referencia el trabajo de Buckley et al [15].

\section{Resultados}

\section{Acerca de la opinión de los alumnos}

Acerca de las ventajas del uso del portafolio, en la mayoría de los casos, los alumnos respondieron que el portafolio les sirvió como guía para comprender los contenidos que se trabajaban en la asignatura. Asimismo, indicaron que facilitó la comprensión global de ésta.

Desde su papel como alumnos indicaron que les permitió demostrar lo aprendido desde su propio trabajo en el portafolio y, a la vez, revisar aquello que no comprendían con ayuda de los profesores durante las tutorías.

Desde la propuesta artística indicaron que la metodología de trabajo les permitió bajar a la reali- dad aquello que muchas veces en los libros no lograban comprender, es decir, que desde el propio trabajo, a partir de las propuestas del portafolio, lograron hacer prácticos muchos contenidos que generalmente quedaban en la teoría. Algunos de sus comentarios fueron:

- 'Genera un conocimiento más profundo, ya que el dibujo origina un proceso interno que fuerza al alumno a desarrollar ideas que en los textos se encuentran escritos, se leen y se entienden, pero no se aprenden'.

- 'Investigar y saber para qué usar los materiales o algunos elementos. Hablar con pacientes, doctores, alumnos, investigar en libros, en Internet. Todas ellas, cosas que sin el portafolio no hubiese hecho'.

- 'Aprender nuevos conceptos, alentar la búsqueda e investigación para interiorizarnos con la prostodoncia a través de la reflexión'.

- 'Me permitió relacionar los trabajos y compararlos, ver su evolución. Entendí lo que me decían cuando me decían «si uno lo sabe dibujar, lo sabe hacer»'.

- 'Me ayudó a resolver dudas. Permitió una consulta constante con los docentes y también me incitó a buscar información para poder realizarlo. Entendí que si uno puede dibujar lo que se le pide, es porque lo ha entendido.'

Estos constituyen solamente algunos ejemplos textuales de los comentarios de los alumnos. Puede destacarse su experiencia desde la reflexión, la investigación, el autoaprendizaje y la relación docentealumno.

Entre los inconvenientes en el uso del portafolio, el más destacado fue en relación al dibujo. La mayoría de los alumnos indicó que fue dificultoso porque no sabían dibujar o porque les resultaba muy trabajoso hacerlo. Asimismo, se destacó el tiempo de trabajo, los alumnos expresaron que fue una actividad muy demandante. Los alumnos también señalaron que anticipar algunos temas que no habían trabajado en clase o que eran nuevos para ellos resultó muy complejo para el trabajo en los distintos documentos que incluía el portafolio. En algunos casos, era costoso interpretar los enunciados y los textos pero, sin embargo, esto obligaba a realizar las propuestas con mayor esfuerzo. Por otro lado, mencionaron que el portafolio no contemplaba todos los contenidos trabajados en la asignatura. Finalmente, el mismo porcentaje de alumnos que indicó que el mayor inconveniente fue la demanda de tiempo de trabajo, señaló no haber tenido inconvenientes. En la figura 1 se indican los porcentajes de 
alumnos que señalaron como inconvenientes las variables más representativas.

Como sugerencias para la mejora del portafolio, los alumnos indicaron la importancia de explicar algunas consignas mediante imágenes o vídeos, incluir más temas, permitir la asistencia a la clínica para detallar las experiencias a través de fotografías, permitir la exposición grupal de cada portafolio, realizar algunas tareas relacionadas con el portafolio en la clase-taller y hacer su entrega obligatoria en los exámenes parciales para comprometer al alumno en su entrega a tiempo.

En cuanto a las preguntas cerradas evaluadas mediante una escala tipo Likert, se obtuvieron los siguientes resultados (se indican los resultados para los que se obtuvo mayor respuesta):

- El 58,45\% de los alumnos respondió estar de acuerdo acerca de que el uso del portafolio contribuyó a su aprendizaje de forma significativa.

- El 67,92\% de los alumnos indicó estar de acuerdo con que el portafolio fue una herramienta de enseñanza.

- El 41,50\% de los estudiantes indicó estar muy de acuerdo en que el portafolio contribuyó a la reflexión acerca de su trabajo académico, lo que había sido expresado en gran parte por los alumnos en alguna de las preguntas de respuesta abierta.

- La respuesta fue neutral para el $54,71 \%$ de los alumnos cuando se les preguntó acerca de si el portafolio había contribuido para un trabajo en equipo.

- El 62,26\% de los alumnos siente que en buena medida se encuentra preparado para comenzar la actividad clínica al siguiente año.

- El 58,49\% de los estudiantes calificó como buena la enseñanza ofrecida en el curso anual 'Prostodoncia I'.

En las figuras 2 y 3 se reflejan los números de respuestas para cada pregunta sobre la base de 53 respuestas totales.

En general, la encuesta confirmó una percepción positiva de los estudiantes acerca de las fortalezas del portafolio. La opinión de los estudiantes, en las encuestas realizadas, expresa en un elevado porcentaje que el portafolio mejoró su aprendizaje de forma significativa y que fue una buena herramienta de enseñanza. Lo más importante fue que contribuyó a la reflexión en su trabajo académico (71\%).

\section{Acerca de la evaluación del profesorado}

Los profesores reconocieron la importancia de un espacio donde primó el acercamiento con el alum-
Figura 2. Opinión de los alumnos en relación al portafolio.

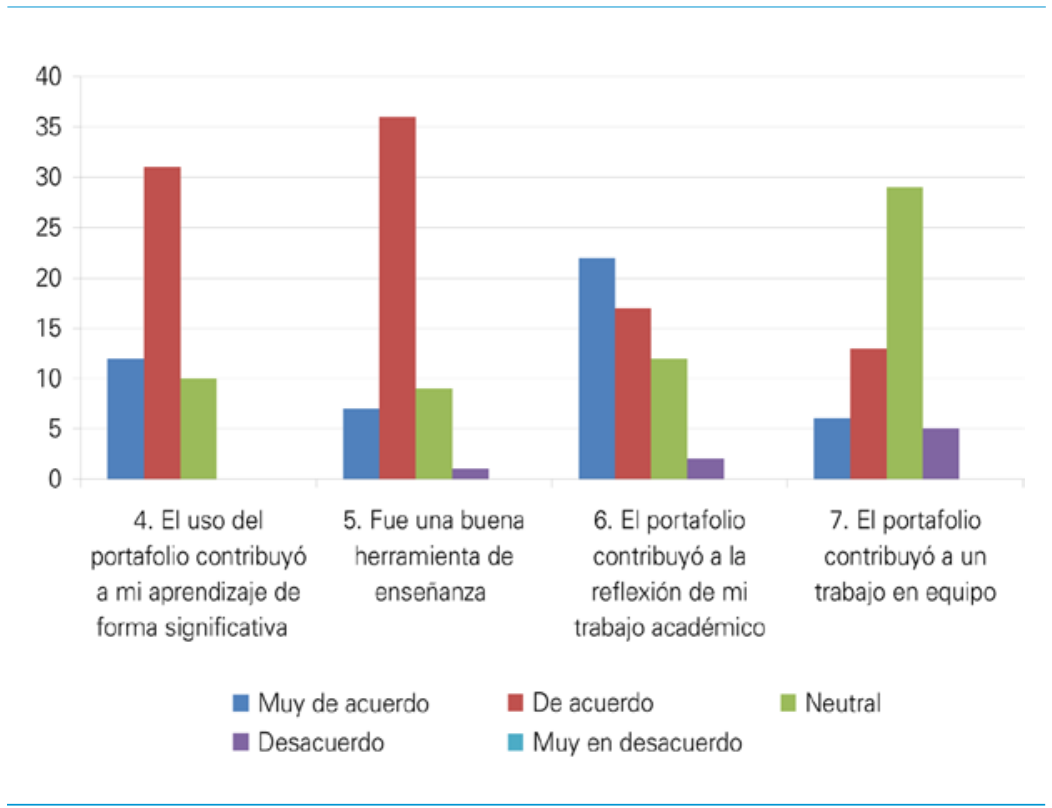

Figura 3. Opinión de los alumnos en relación a la actividad docente desarrollada.

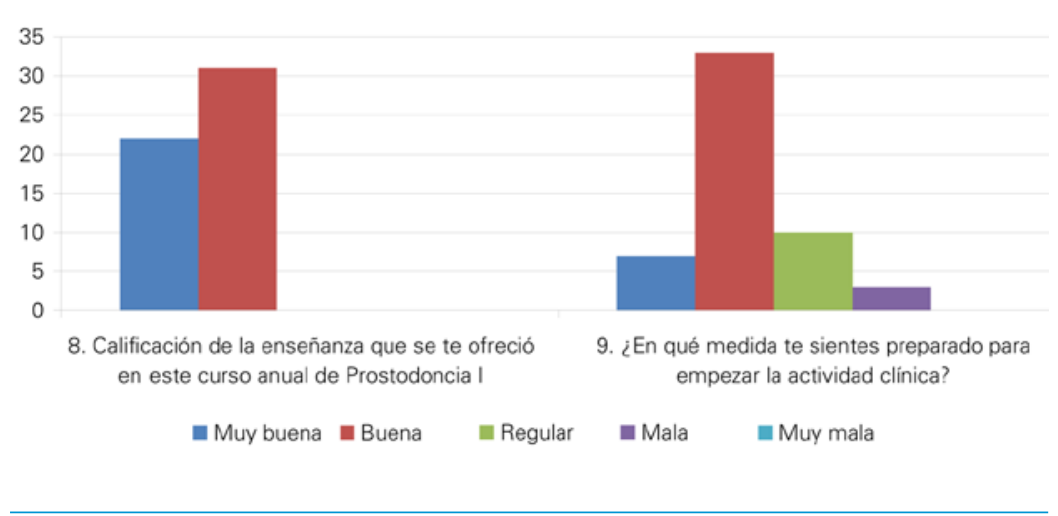

no. El análisis de las producciones durante las tutorías requirió un momento de contacto personal con cada uno de los ellos. Esto permitió conocer a los estudiantes en particular y realizar un análisis más profundo con cada uno acerca de sus producciones y su relación con los contenidos de la asignatura.

Tras el análisis de las producciones de los estudiantes, los profesores percibieron cuáles eran los contenidos que generaban mayor conflicto en el proceso de enseñanza y aprendizaje, se comprobó la mejora en el aprendizaje de diversos contenidos por parte de los alumnos, se identificó a los alumnos de 
bajo rendimiento y el feedback de cada producción se realizó de forma inmediata.

El trabajo permanente con los alumnos a través de las tutorías posibilitó a los alumnos reflexionar acerca de las tareas que realizaban, evaluar el proceso del que eran participes, modificar la actitud memorística tan presente en la rehabilitación oral por medio de la enseñanza de técnicas y procedimientos, y comprender la relación entre lo que se enseña en el taller y su futura aplicación en la clínica. Esto se vio reflejado en la encuesta, con un alto porcentaje en la respuesta referida a esta variable.

Por su parte evaluaron como inconvenientes que su implementación se hace dificultosa en cohortes numerosas y que el número de documentos de cada portafolio no debe ser extenso, que el abordaje del portafolio y la lectura de sus documentos requirió un tiempo y esfuerzo mayores de los habituales y que su uso excesivo podría aburrir al alumno por el tiempo que le toma de otras actividades que son más de su interés.

\section{Discusión}

En primer lugar, es destacable el encuentro de un espacio de comunicación e intercambio con el alumno. Asimismo, este acercamiento con el alumno a través de la realización del portafolio permitió reencontrar un lugar de encuentro que en cierta medida, en asignaturas como 'Prostodoncia I', se había perdido; es decir, se recuperó el espacio de relación alumno-docente protegido de las interrupciones, donde el conocimiento mutuo y la discusión compartida, más allá del saber sobre la asignatura, aportó una maravillosa consecuencia de reafirmación del ser docente.

En segundo lugar, el análisis de los documentos permitió identificar los temas conflictivos y comprobar la evolución en el conocimiento de los alumnos, reconociendo diferencias en cuanto a los rendimientos y pudiendo en todos los casos entregar una devolución inmediata.

Otro punto destacable en la implementación del portafolio, también mencionado por los alumnos en las encuestas, fue la posibilidad de reflexión acerca de la propia tarea del estudiante y del proceso del que son partícipes, modificando la tendencia hacia el aprendizaje memorístico, tan presente en la rehabilitación oral debido a la preeminencia de ciertas técnicas y procedimientos en la enseñanza, y demostrando que existe una relación muy importante entre lo que se enseña en el taller y su futura implementación en la clínica. Esto quiere decir que hubo un cambio de actitud frente al aprendizaje por parte del alumno.

Como indica Fenstermacher [16], la tarea del profesor consiste en apoyar el deseo del alumno de 'estudiantar', de ser estudiante, y mejorar así su capacidad. El profesor más bien instruye al estudiante sobre cómo adquirir el contenido a partir de sí mismo. A medida que el estudiante es capaz de adquirir el contenido, aprende, y esto es lo que se vio reflejado en la implementación del portafolio como experiencia educativa.

Como quedó demostrado, cuando los alumnos comprenden claramente los criterios expuestos en él, el portafolio les permite dirigir sus trabajos y optimizar sus desempeños. De ese modo, su aplicación coincide con el criterio actual de mejorar las herramientas de evaluación, ya que además de poder observar el desempeño de los alumnos, les sirve a ellos para su propio aprendizaje.

En cuanto a los inconvenientes, y más allá de aquellos enunciados por los alumnos en las encuestas o de los reconocidos en la bibliografía, puede afirmarse que las consignas deben ser claras y concisas para no perder el interés del alumno por la tarea.

Por último, se valida la decisión de incorporar el dibujo en el desarrollo de los documentos como un recurso de utilidad para la disciplina de la prostodoncia en el nivel inicial. Si bien se reconoce la dificultad para la técnica por parte de los alumnos, el esfuerzo de éstos dio como resultado notables avances en su conocimiento acerca de la asignatura.

En conclusión, como investigación educativa en acción [17], esta experiencia ha cumplido con el propósito de reflexionar acerca del portafolio, buscando resolver sus interrogantes en contacto con los alumnos y en discusión con los profesores, realizando un análisis inmediato dentro de la propia institución, demostrando a través de su instrumentación ser una metodología para mejorar las prácticas y para generar conocimientos desde las acciones o intervenciones de los docentes y alumnos. Los resultados obtenidos del análisis demostraron que el portafolio se considera una herramienta que permite enriquecer las prácticas educativas, generando conocimientos desde las construcciones propias de los alumnos, haciéndolas conscientes en el proceso de enseñanza y aprendizaje. El portafolio se convierte así en una herramienta poderosa para:

- Formar profesores reflexivos.

- Recuperar las propias concepciones de enseñanza y de aprendizaje.

- Generar nuevos y mejores espacios de comunicación entre profesores y alumnos. 
- Valorar la narrativa y la creatividad como variables importantes en la construcción del conocimiento.

- Reconocer el aprendizaje como una construcción colaborativa.

Los resultados de la aplicación del portafolio fueron muy positivos y respaldan la percepción inicial que estuvo en el origen de la experiencia acerca de su utilidad. Ha resultado ser una herramienta válida, aceptable, subsistente y de gran ayuda para la formación preclínica del graduando en odontología.

El portafolio, como estrategia valiosa al ser utilizada moderadamente, se convierte en un proceso de investigación tanto para el docente como para el alumno. Al docente, le posibilita indagar sobre la adecuación de sus propuestas de enseñanza, mejorando así sus tareas y, con ello, el proceso de formación de los odontólogos. El alumno, por su parte, puede investigar sobre sus procesos cognitivos: sobre cómo aprende, qué jerarquiza y cómo reflexiona. Por tanto, no es sólo una herramienta didáctica que utiliza el docente, sino también una herramienta de aprendizaje para el alumno.

$\mathrm{Su}$ aplicación coincide con el criterio actual de mejorar las herramientas de evaluación porque, además de poder observar el desempeño de los alumnos, les sirvió a ellos para su aprendizaje.

Fue un recurso que permitió llevar adelante, incorporar a nuestro quehacer, una mejor evaluación formativa. Se pudo identificar en esta experiencia los procesos que, según Camillioni [14], la evaluación formativa requiere, especialmente la recogida de datos sobre el rendimiento de los alumnos, la selección de los métodos a utilizar consistentes en la observación del comportamiento del alumno mientras realiza la tarea y mientras reflexiona, llevar el registro de lo observado, y la adaptación de las estrategias de enseñanza y aprendizaje en atención a la información recogida y a su interpretación por el profesor.

Quedó evidenciado que cuando los alumnos comprenden claramente los criterios expuestos en él, el portafolio se convierte en una herramienta que les permite dirigir su trabajo y mejorar su desempeño.

Una evaluación por portafolio, en sí, requiere un gran esfuerzo y trabajo por parte del profesor al recoger información tan variada acerca del trabajo del alumno, de la tarea docente, de los contenidos de una asignatura determinada. Sin embargo, si se observa que este tipo de evaluación se integra y se de- sarrolla a lo largo del dictado de una asignatura, son más los beneficios que la acompañan que las dificultades a las que está sujeta. Cabe destacar que el esfuerzo que requiere realizar un portafolio con cierto número de alumnos se equipara a los grandes resultados que se obtienen a partir del feedback continuo con el alumnado.

\section{Bibliografía}

1. Wilkinson TJ, Challis M, Hobma SO, Newble DI, Parboosingh JT, Sibbald RG, et al. The use of portfolios for assessment of the competence and performance of doctors in practice. Med Educ 2002; 36: 918-24.

2. Schön, D La formación de profesionales reflexivos. Hacia un nuevo diseño de la enseñanza y el aprendizaje en las profesiones. Madrid: Paidós-MEC; 1992.

3. Barberà E. Evaluación por portafolio en la universidad. Forum sobre docencia universitaria de la Universitat de Barcelona (2006). URL: http://www.ub.edu/forum/Catalan/welcome.htm. [10.06.2011].

4. Klenowski V. Desarrollo de portafolios para el aprendizaje y la evaluación. Madrid: Narcea; 2005.

5. Barragán R. El portafolio, metodología de evaluación y aprendizaje de cara al nuevo Espacio Europeo de Educación Superior. Una experiencia práctica en la Universidad de Sevilla. Revista Latinoamericana de Tecnología Educativa 2005; 4: 121-39.

6. Villalobos J. Portafolios y reflexión: instrumentos de evaluación en una clase de escritura. Educere 2002; 5: 390-6.

7. Chacón CT, Chacón-Corzo MA. El uso del portafolio en la enseñanza de lenguas extranjeras. Acción Pedagógica 2011; 20: 32-41.

8. Agra MJ, Gerwerc A, Montero L. El portafolios como herramienta de análisis en experiencias de formación online y presenciales. Enseñanza: Anuario Interuniversitario de Didáctica 2003; 21: 101-14.

9. Romero-López MA, Crisol-Moya E. El portafolio, herramienta de autoevaluación del aprendizaje de los estudiantes. Una experiencia práctica en la Universidad de Granada. Revista de Docencia e Investigación 2011; 21: 25-50.

10. Universidad Nacional Autónoma de México. URL: http://www. cuaed.unam.mx/boletin/boletinesanteriores/boletinsuayed17/ portafolio.php.

11. Blanco A. Desarrollo y evaluación de competencias en educación superior. Madrid: Narcea; 2009.

12. Edwards B. Nuevo aprender a dibujar con el lado derecho del cerebro. Barcelona: Urano; 2008.

13. McLean M, Henson Q, Hiles L. The possible contribution of student drawings to evaluation in a new problem-based learning medical programme: a pilot study. Med Educ 2003; 37: 895-906.

14. Camilloni A. Sobre la evaluación formativa de los aprendizajes. Quehacer Educativo 2004; 68: 6-12.

15. Buckley S, Coleman J, Davison I, Khan KS, Zamora J, Malick S, et al. The educational effects of portfolios on undergraduate student learning: a Best Evidence Medical Education (BEME) systematic review. BEME Guide No. 11. Med Teach 2009; 31: 282-98.

16. Fenstermacher $\mathrm{G}$. Tres aspectos de la filosofía de la investigación sobre la enseñanza. In Wittrock M, ed. La investigación en la enseñanza. Tomo 1. Madrid: Paidós-MEC; 1989.

17. Sverdlick I, comp. La investigación educativa, una herramienta de conocimiento y de acción. Buenos Aires: Noveduc; 2010. 